\title{
Simulierte Realität
}

\section{Erhard Taverna}

Dr. med., Mitglied der Redaktion

Die traditionelle chirurgische Ausbildung findet im Operationssaal statt. Übungen an der Leiche oder an Tierkadavern spielen eine untergeordnete Rolle. Mit Einzug minimalinvasiver Techniken in den frühen 1980er Jahren stieg der Bedarf an alternativen Trainingsmöglichkeiten für den Nachwuchs. Es dauerte beinahe 20 Jahre, bis die rasant fortschreitende Computertechnologie die Entwicklung von Simulatoren erlaubte, welche es ermöglichen, die Kompetenzen des Chirurgen stressfrei, risikolos und am lebensnahen Modell mit Originalinstrumenten zu steigern. Heutige Simulatoren, ähnlich jenen in der Flugzeugindustrie, erlauben standardisierte Abläufe,

\section{Es herrscht eine entspannte und dennoch arbeitsintensive Atmosphäre im Technologie- betrieb.}

beliebige Wiederholungen, Bewertungen, Dokumentation sowie selbständiges Lernen unter Anleitung der Software in Kombination mit einer optimierten Unter- stützung durch erfahrene Kollegen. Fortgeschrittene Modellierungen und Grafikanimationen ermöglichen das Erstellen von einfachen bis komplexen realisti-

Das Entwicklungsteam und die Produkte gehören weltweit zur ersten Liga dieser Branche.

schen Trainingsmodulen für das Grundlagentraining sowie das Üben von Routine- bis hin zu selteneren Eingriffen.

Raimundo Sierra macht es am Patientenmodell vor. Paradebeispiele im Schauraum seiner Firma VirtaMed sind das Kunststoffmodell eines Hüft-, Knie- und Schultergelenks. Durch die vorgegebenen Inzisionsstellen dringt er in das Gelenk ein. Die linke, weniger dominante Hand hält das Endoskop mit der Kamera, die rechte, dominante, sucht mit der Greifzange den richtigen Weg durch das Gewebe. Das beidhändige Navigieren im dreidimensionalen Raum unter Bildschirmkontrolle erinnert an ein Computerspiel. Ein

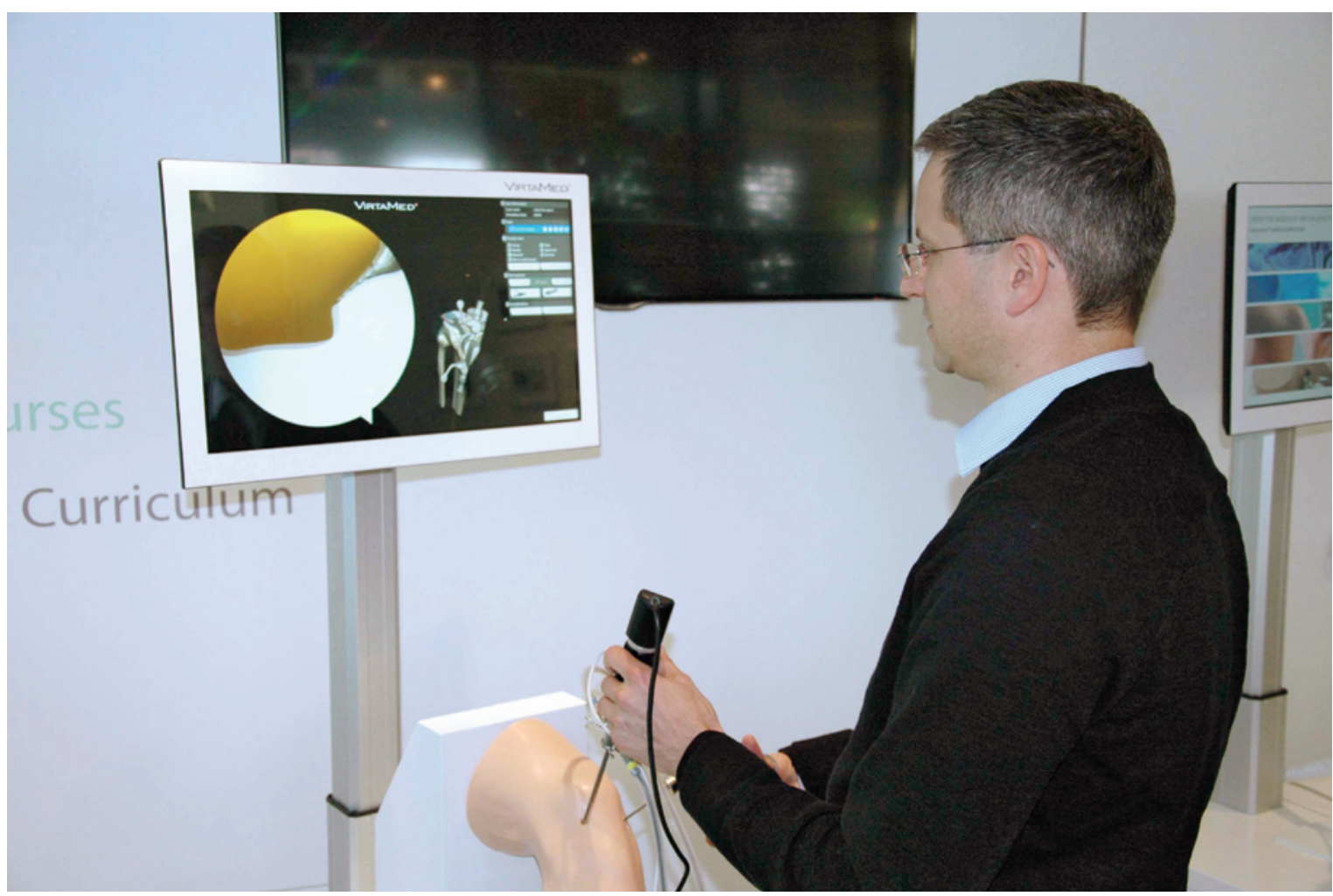

Raimundo Sierra. 
gelber Stern ist optisch zu zentrieren, mit der Greifzange zu fassen und zu entfernen, äquivalent zum Entfernen von losen Gewebeteilen. Was spielerisch beginnt, erfordert zunächst nur räumliche Orientierung und Geschicklichkeit. Ein Vorspiel im didaktischen Aufbau, der Schritt für Schritt den Probanden zu diagnostischen und therapeutischen Herausforderungen, inklusive Komplikationen, heranführt. Dank der optimalen Kombination der Software mit Sensoren sowie Knochenmodellen ist eine taktile Rückkoppelung möglich, neue Materialien perfektionieren die haptische Illusion. Die Grundausrüstung eines Simulators kann für endoskopische Eingriffe verschiedener modular kombinierter Disziplinen eingesetzt werden.

Ausgehend vom einem Nationalen Forschungsprojekt mit Schwerpunkt «simulierte Chirurgie» entwickelten der ETH-Ingenieur sowie weitere 15 Doktoranden von 2001 bis 2007, in Zusammenarbeit mit der Gynäkologie des USZ, einen Hysteroskopiesimulator. Dem folgten später Patientenmodelle für die Urologie und die Orthopädie. 2007 wird der Spin-off VirtaMed gegründet und von Stefan Tuchschmid geführt. Seit 2014 führen Sierra und Tuchschmid als gleichberechtigte Partner ein Unternehmen, welches heute über 70 Mitarbeiter beschäftigt und im Ranking der schweizeri-

\section{Rund die Hälfte der hier entwickelten Produkte wird zu Demonstrationszwecken oder für Trainingsangebote angeschafft, die andere Hälfte ist klinisch im Einsatz.}

schen Handelszeitung als Wachstumschampion 2017 aufgelistet wurde. Die Software- und Hardwareentwickler haben sich in ihren zahlreichen Arbeitsräumen über ein ganzes Stockwerk ausgedehnt. Es herrscht eine entspannte und dennoch arbeitsintensive Atmosphäre im Technologiebetrieb nahe des Bahnhofs Schlieren. Hochqualifizierte junge Menschen verschiedener Nationen verständigen sich in der Universalsprache Englisch. Das Entwicklungsteam und die Produkte gehören weltweit zur ersten Liga dieser Branche. Für die Entwicklung unentbehrlich ist eine optimale Vernetzung mit Universitätskliniken im In- und Ausland, Forschungszentren und Fachgesellschaften. Das schweizerische Examen zur Erlangung des Facharzttitels für Orthopädie und Traumatologie des Bewegungsapparates wird teilweise auf dem Arthroskopie-Simulator VirtaMed ArthoS abgelegt. «Swiss en-

\section{In der ganzen Medizin ist ein Kulturwandel im Gange.}

gineering» lockte auch die American Society for Reproductive Medicine nach Zürich. Am Embryo Transfer Simulator lässt sich beispielsweise die heikle Einführung der Sonde in den Zervikalkanal üben, was die Erfolgsrate bei Fruchtbarkeitsbehandlungen bei Invitro-Fertilisation wesentlich verbessern kann. Auch Einfacheres, wie das Einbringen einer Spirale, gehört $\mathrm{zu}$ den weiteren Anwendungsmöglichkeiten. Längst assistiert im OP auch die Technik. Der vielzitierte $D a$ Vinci-Roboter fusst auf dem ähnlichen Prinzip einer Steuerkonsole, ergänzt mit einem erweiterten Videosystem, das eine volle, dreidimensionale Sicht ermöglicht.

Rund die Hälfte der hier entwickelten Produkte wird von Medizinaltechnikfirmen zu Demonstrationszwecken sowie für deren Trainingsangebote angeschafft, die andere Hälfte ist klinisch im Einsatz. Etwa 98\% des Umsatzes werden im Ausland generiert. Startup, ein Magazin der Handelszeitung, publizierte 2016 eine Hitparade der 100 vielversprechendsten Hightechunternehmen in der Schweiz. Rund ein Viertel davon betrifft den medizinischen Bereich. Raimundo Sierra ist längst über diese Phase hinausgewachsen. Soeben aus China zurückgekehrt, sind er und sein Team auf Expansionskurs. In der ganzen Medizin ist ein Kulturwandel im Gange. Das alte Lehrer-Schüler-Verhältnis gilt immer noch, wenn auch zunehmend ergänzt und bereichert durch die wachsenden Möglichkeiten der Virtual Reality.

Bildnachweis Erhard Taverna

Weiterführende Informationen unter: www.virtamed.com 